\title{
Inflammation and the Blood Microvascular System
}

\author{
Jordan S. Pober ${ }^{1}$ and William C. Sessa ${ }^{2}$ \\ ${ }^{1}$ Department of Immunobiology, Yale University School of Medicine, New Haven, \\ Connecticut 06520-8089 \\ ${ }^{2}$ Department of Pharmacology, Yale University School of Medicine, New Haven, \\ Connecticut 06520-8089 \\ Correspondence: jordan.pober@yale.edu
}

Acute and chronic inflammation is associated with changes in microvascular form and function. At rest, endothelial cells maintain a nonthrombogenic, nonreactive surface at the interface between blood and tissue. However, on activation by proinflammatory mediators, the endothelium becomes a major participant in the generation of the inflammatory response. These functions of endothelium are modified by the other cell populations of the microvessel wall, namely pericytes, and smooth muscle cells. This article reviews recent advances in understanding the roles played by microvessels in inflammation.

nflammation, a major component of the innate immune response, is typically a local process historically characterized by cardinal features, such as rubor (redness) and calor (warmth), both caused by increased blood flow to the inflamed site, and tumor (swelling), caused by extravasation of fluid, plasma proteins, and leukocytes (changes attributable to actions of the local microvasculature). In this article, we will review how blood microvessels and the cells from which they are formed respond to innate inflammatory signals, leading to inflammation. We will not discuss recently reviewed topics, such as the lymphatic circulation (Alitalo 2011) or role of blood vessels in adaptive immunity (Pober and Tellides 2012). We begin with an overview of the blood microvascular system.

\section{ORGANIZATION OF THE BLOOD MICROVASCULAR SYSTEM}

The blood vascular system consists of two (systemic and pulmonary) closed loops organized into distinct segments. Large elastic arteries arising from the heart give rise to smaller muscular arteries that convey blood to specific organs. Within the organs, arteries further arborize, ending as arterioles having diameters in tens of micrometers. Arterioles, the most proximal segments of the microvasculature, are internally lined by a monolayer of endothelial cells (ECs), connected to each other by intermixed tight and adherens junctions, that is invested by circumferentially arranged layers (lamellae) of vascular smooth muscle cells (SMCs). ECs are separated from the SMC layers by both a condensed layer

Editor: Ruslan M. Medzhitov

Additional Perspectives on Innate Immunity and Inflammation available at www.cshperspectives.org

Copyright (C) 2015 Cold Spring Harbor Laboratory Press; all rights reserved; doi: 10.1101/cshperspect.a016345

Cite this article as Cold Spring Harb Perspect Biol 2015;7:a016345 
of extracellular matrix enriched in type IV collagen and laminin, known as basement membrane (BM), and deep to the BM, by a thick connective tissue band enriched in elastin fibers called the internal elastic lamina (IEL). The IEL marks the boundary between the arterial intima and the media. The intima between the BM and IEL is formed by a small (in arterioles) zone of loose connective tissue that may contain both SMCs and leukocytes. Focal discontinuities in both BM and the IEL allow ECs, which are electrically coupled to each other through gap junctions, to form gap junctions with underlying SMCs (Behringer and Segal 2012). A less welldefined outer elastic lamina marks the outer boundary of the media, separating it from the parenchyma of the surrounding organ.

At their distal end, arterioles arborize further to give rise to capillaries. This portion of the microvasculature varies widely in structure from tissue to tissue but has the general feature of being formed by a series of single ECs, each of which curves around to form a lumenized tube with diameters of $<10 \mu \mathrm{m}$. Capillaries are very numerous and comprise the major surface for exchange of gases, fluid, and nutrients between blood and tissue. Heterogeneity of capillary ECs affects the degree to which they limit or permit such exchanges (Aird 2007a,b). Proteins may pass either through the junctions between capillary ECs (paracellular transit) or through the ECs themselves (transcellular transit via vesicles, channels, or fenestrae). Highly impermeant capillaries, such as those in the central nervous system (CNS), form many more tight junctions between adjacent ECs than other capillaries, limiting paracellular passage of proteins, and lack fenestrae and channels, forcing all exchanges through a limited vesicular transport system. At the other extreme, sinusoidal capillaries in liver, spleen, or bone marrow have open gaps between adjacent ECs. Some capillary ECs form fenestrae (holes) that allow transcellular passage of proteins. Fenestrae may be filled in by organized protein structures called diaphragms, partially limiting protein transit. Capillaries are invested and supported by a single discontinuous layer of contractile cells known as pericytes (PCs) (Dore-Duffy and Cleary 2011). PCs are arrayed longitudinally along the capillary and each PC may contact multiple ECs. The ratio of PCs to ECs varies among different tissues, being highest at 1:1 in the CNS where permeability is the lowest. Unlike SMCs of arterioles that form their own layers of extracellular matrix typically enriched for type I collagen and elastin, PCs reside within the collagen IV- and laminin-rich BM of the ECs. PCs lack tight junctions but may limit extravasation of plasma proteins indirectly through influence on EC junctions and/or BM composition (Goddard and Iruela-Arispe 2013).

At their termini, capillaries converge and drain their contents into larger caliber vessels known as venules. Postcapillary venules further converge into larger collecting vessels, ultimately connecting the microvasculature to veins that drain blood from the organ and return it to the heart. The EC lining of larger venules and veins is invested by layers of vascular SMCs, although these are typically less well organized into distinct lamellae and are fewer in number than those found in arterioles and the IEL is less well formed. In contrast, postcapillary venules, like capillaries, are primarily invested by PCs rather than SMCs. The ECs of the postcapillary venules are connected to each other by adherens junctions and lack tight junctions (Goddard and Iruela-Arispe 2013), making them more intrinsically leaky than the continuous capillaries that empty into them (Rous and Smith 1931). The capillaries themselves may be leakier at their venular end than they are at the arteriolar origin. In the skin, the BM of postcapillary venules has a distinct appearance by transmission electron microscopic, forming lamellae as opposed to the more homogeneous BM of arterioles and capillaries (Braverman 1989), implying a difference in composition and/or organization that may influence EC functions.

The embryological origin of the three principal cell types of the microvasculature are distinct. Almost all ECs in the adult organism can trace their lineage back to angioblasts arising in the blood islands of the aorto-gonadal-mesonephric region that migrate and differentiate into endothelial progenitor cells (EPCs) and/ or fully differentiated ECs. Consequently, the 
tissue-specific features of capillary ECs develop in response to local environmental cues rather than to distinct embryological origins (Aird 2007a,b). The formation of new blood vessels in settings of chronic inflammation begins with outgrowth and replication of differentiated EC lining preexisting microvessels, although EPCs, probably residing within the vessel wall, may also contribute. Vascular SMCs of large vessels are more heterogeneous in their origin. Some, as in the proximal aorta, arise from the second heart field or neural crest, whereas others develop from local mesenchyme, often maintaining embryological patterns of Hox gene expression (Majesky 2007). Both SMCs and PCs of the microvasculature are probably derived from local mesenchyme but SMCs and PCs within the CNS may derive from neural crest and, at some sites, PCs may derive from ECs undergoing "endothelial to mesenchymal cell transition" (Armulik et al. 2011). Little is known about tissuespecific differences among PCs and how these may affect the contributions of these mural cells to inflammatory processes. Mesenchymal stem cells (MSCs, also called mesenchymal stromal cells) localize to the PC layer of microvessels (Crisan et al. 2008), but it is not clear whether all PCs have multipotency.

\section{HOMEOSTATIC FUNCTIONS OF THE VASCULATURE}

The vascular system under normal circumstances performs four major homeostatic functions: (1) it keeps blood fluid; (2) it regulates perfusion of different organs; (3) it prevents inappropriate activation of leukocytes; and (4) it regulates permselective exchange of macromolecules between blood and tissues. The prevention of coagulation is a general feature of the whole vascular system and will be discussed here. The other homeostatic properties of the vasculature are typically assigned to specific microvascular segments and will be discussed in subsequent sections.

ECs prevent intravascular coagulation by several mechanisms. First, ECs sequester phosphatidylserine (PS) to the inner leaflet of their plasma membrane, depriving circulating clot- ting factors of a surface required for assembly into functional complexes. Second, resting ECs express proteins that inhibit coagulation, notably tissue factor pathway inhibitor (TFPI) and thrombomodulin. TFPI prevents tissue factors from capturing and accelerating the catalytic activity of factor VIIa, the initiator of the intrinsic coagulation cascade. Thrombomodulin captures active thrombin and alters its substrate specificity from a procoagulant protease that converts fibrinogen to fibrin to an anticoagulant protease that cleaves and activates protein C. Activated protein C, when bound to its receptor on ECs and in combination with protein $\mathrm{S}$ (made by ECs among other cell types), cleaves and inactivates various coagulation factors, such as factor V (Bouwens et al. 2013). Third, ECs also express proteoglycans bearing heparin sulfate glycosaminoglycans (GAGs) that capture and activate antithrombin III, creating a substrate trap for active thrombin. Fourth, resting ECs synthesize plasminogen activators (both tissue type and urokinase type) as well as the receptor for urokinase-type plasminogen activator, converting circulating plasminogen to plasmin, a protease that cleaves fibrin and lyses incipient thrombi. Fifth, ECs prevent platelet activation by inhibiting thrombin, by preventing contact with $\mathrm{BM}$ or interstitial collagens, and by degrading extracellular adenosine- $5^{\prime}$-triphosphate (ATP). Resting ECs synthesize von Willebrand factor (vWf), a platelet adhesive molecule important for platelet adhesion in settings of high shear stress, but sequester it internally within storage granules known as WeibelPalade bodies (WPB) where it is inaccessible to platelets. Finally, resting ECs synthesize and release small quantities of PGI2 and nitric oxide (NO) sufficient to inhibit platelet activation by raising intracellular cAMP and cGMP, respectively. Inhibition of platelet activation by these mediators may act synergistically.

\section{ARTERIOLES AND CONTROL OF BLOOD FLOW}

The major roles of arterioles are to control pressure, flow, and nutrient delivery to the capillary beds. Arborizing branches of arterioles provide 
a physical buffer or resistance to normalize pressure gradients generated throughout the cardiac cycle. As pressure increases into proximal arteries during cardiac contraction, distal arterioles will vasoconstrict to limit pressure and flow into the microcirculation through a mechanism called myogenic vasoconstriction. This arteriole, smooth muscle intrinsic response is critical for maintaining constant flow to vital organs, such as the brain, kidney, and heart and serves as a mechanism for reducing pressure into more structurally fragile capillary beds. In addition to myogenic constriction in arterioles, ECs in arteries and arterioles can sense changes in flow and release local autacoids, such as NO, lipid metabolites, and other mediators to increase or decrease vessel diameter. During acute inflammatory responses, leukocyte-derived mediators such histamine and bradykinin will cause arteriolar dilation thereby increasing blood flow leading to rubor. Histamine-induced arteriolar dilation is abrogated in mice lacking the endothelial nitric oxide synthase gene (eNOS) (Payne et al. 2003); however, other vasodilatory mediators, such as PGI2 and endothelium-derived hyperpolarization factors may contribute to enhanced blood flow in response to proinflammatory molecules. The increase in flow and pressure will increase intravascular hydrostatic pressure providing a gradient for the extravasation of fluid and protein through postcapillary venules into tissue. This occurs simultaneously with retraction of EC lining the venules as highlighted below.

\section{POSTCAPILLARY VENULES, PLASMA PROTEIN EXTRAVASATION, AND LEUKOCYTE RECRUITMENT}

In their basal state, venular ECs form a barrier sufficient to retain most proteins, including albumin (Dejana and Giampietro 2012). The oncotic effect of these plasma proteins serves to limit the extravasation of fluid. Resting ECs also fail to recruit leukocytes, largely attributed to the absence of luminal molecules capable of capturing these cells from the circulation. Similarly, leukocyte-activating chemokines are also absent on resting ECs. However, certain leukocyte adhesion molecules (e.g., P-selectin [CD62P]) and some chemokines (e.g., interleukin [IL]-8 [CXCL8], MCP-1 [CCL2], and eotaxin 3 [CCL26]), are expressed in resting ECs; they are sequestered within WPB and, thus, unavailable to circulating leukocytes (Rondaij et al. 2006). Furthermore, basal NO production in ECs can potentially exert anti-inflammatory effects by inhibiting activation of leukocytes.

Changes in venular ECs alter the behavior of these cells to promote inflammation; such changes have been denoted as type I and/or type II activation (Pober and Sessa 2007). Many mediators of type I activation are vasoactive autacoids, such as histamine, that signal through G-protein-coupled receptors (GPCRs). Histamine receptors are more highly expressed on venular ECs than they are on arteriolar or capillary ECs (Heltianu et al. 1982), although histamine can stimulate vasodilator production from arteriolar ECs. The EC signaling pathways activated by these mediators have been reviewed elsewhere (Pober and Sessa 2007). Venular EC responses to histamine include: transient contraction of ECs, creating intercellular gaps and leading to parcellular escape of plasma proteins (Majno et al. 1961); regulated exocytosis of the contents of WPB, bringing molecules, such as vWf, P-selectin, and certain stored chemokines (IL-8, MCP-1, eotaxin-3) to the luminal cell surface (Lorant et al. 1991); and synthesis of lipid mediators, such as platelet-activating factor (PAF), a potent activator of leukocytes as well as platelets. Coexpression of leukocyte-binding proteins (P-selectin) and leukocyte-activating molecules (PAF, chemokines) on the EC plasma membrane has been described as a "juxtacrine" signaling that, in vitro, can capture and stimulate the transendothelial extravasation of neutrophils (Lorant et al. 1991). However, GPCR-induced signals are self-limited in duration to $\sim 15$ min and the same signals induce synthesis of other mediators that inhibit leukocyte activation, such as NO. Consequently, the result of histamine injection is simply a transient vasodilation and vascular leak ("wheal and flare") that quickly resolves without significant leukocyte recruitment. 
Sustained tissue swelling and significant leukocyte recruitment is dependent on type II activation of venular EC, mediated by inflammatory cytokines. The prototypical examples of such cytokines are IL- 1 (both IL- $1 \alpha$ and IL-1 $\beta$ ) and tumor necrosis factor (TNF, often designated as TNF- $\alpha$ ). ECs express both the signaling IL-1 receptor (IL-1R1, designated CD121a) and, in the resting state, TNF receptor 1 (TNFR1 or CD120a) but not TNFR2 (CD120b) (Al-Lamki et al. 2005). The binding of IL-1 or TNF to these receptors activates transcription factors, thereby increasing expression of mRNAs encoding proteins that capture and activate leukocytes. For example, TNF or IL-1 induce de novo expression of leukocyte adhesion molecules E-selectin (CD62E) and vascular cell adhesion molecule (VCAM)-1 (CD104) and increase expression of intercellular adhesion molecule (ICAM)-1 (CD54) from a low but detectable basal state (Pober and Sessa 2007). In mice, but not humans, P-selectin transcription is induced as well (Pan et al. 1998). TNF also induces miRs that feedback and limit E-selectin and ICAM-1 expression (Suarez et al. 2010). TNF and IL-1 also induce synthesis of chemokines, notably of IL- 8 and MCP-1, the principal human chemokines that activate neutrophils and monocytes, respectively. The de novo synthesis of these chemokines is independent of the release of prestored chemokines from WPB, a type I activation responses, as inflammatory cytokines do not cause WPB exocytosis (Zavoico et al. 1989). Chemokines released from EC or from leukocytes within the perivascular space bind to GAG expressed on the EC luminal surface (Mortier et al. 2012), which, along with leukocyte adhesion molecules, provide sustained juxtacrine signaling that leads to leukocyte infiltration in vivo. Fractalkine (CX3CL-1) represents a variation on this theme in that the chemokine moiety that interacts with its receptor, predominantly expressed on a subset of monocytes, is synthesized and expressed as the amino-terminal domain of an EC proteoglycan (Imaizumi et al. 2004). Finally, it should be noted that, because the signaling pathways activated by IL-1 binding to its receptor are also activated by Toll-like receptors (TLRs), with the exception of TLR3, and because human vascular EC express TLR1, TLR2, TLR4, TLR6, and TLR9, engagement of these receptors by appropriate ligands, typically one or more pathogenassociated molecular patterns or damage-associated molecular patterns, can produce type II activation responses (Opitz et al. 2007).

The general model for leukocyte recruitment by type II-activated venular ECs involves a multistep cascade (Ley et al. 2007). In humans, E-selectin typically mediates the initial tethering of the circulating neutrophil or monocyte by recognizing specific carbohydrate determinants, which in the case of neutrophils are attached to L-selectin expressed on the tips of microvillous projections. These adhesive interactions are of low affinity but are rapidly formed. Flowing blood pushes the tethered leukocyte, breaking selectin-mediated attachments that subsequently rapidly reform with new Eselectin molecules as the leukocyte is displaced in the direction of flow, resulting in leukocyte rolling. Monocytes and some $\mathrm{T}$ cells may instead (or additionally) be tethered and roll using leukocyte integrin VLA-4 (CD49d/CD29), that in its low affinity state, can also rapidly form weak attachments to endothelial VCAM1. Some birectional signals may be transmitted during these adhesive interactions, but these are generally thought to provide insufficient activation of the leukocyte to progress to the next step in the cascade, known as firm adhesion. Instead, specific GPCRs on the rolling leukocyte must encounter a cognate ligand, typically an EC-bound chemokine, resulting in signaling that causes the leukocyte to increase affinity of its integrins for EC ligands, specifically LFA-1 (CD11a/CD18) and Mac-1 (CD11b/CD18) for ICAM-1 or VLA-4 (CD49d/CD29) for VCAM-1, changing from a round cell to one spread out on the surface of the ECs. These "firmly adherent" leukocytes acquire motility and use these same integrins and EC ligands to crawl toward EC junctions. At or near the junctions, the EC may project its luminal plasma membrane upward, forming an "adhesion cup" or "docking structure" that partially engulfs the bound leukocyte and within which EC adhesion molecules, specifically ICAM-1 and VCAM-1, cluster (Bar- 
reiro et al. 2004). The clustering of ICAM-1 and possibly VCAM-1 induces the membrane remodeling to occur at the EC junction, facilitating a path for transendothelial migration. At the same time, the leukocyte projects cytosolic processes ("invadosomes") that push between or through the ECs, initiating the process of extravasation (Carman et al. 2007).

Transit across the EC lining of the venule involves sequential molecular interactions with platelet/endothelial cell adhesion molecule PECAM-1 (CD31) and CD99, each of which forms homophilic adhesions with the same molecules expressed on the leukocyte (Muller 2011). VEcadherin and certain tight junction proteins (e.g., junctional adhesion molecule [JAM] A or C) on the EC may also play a role at this stage. Unlike ICAM-1 and VCAM-1, the total levels of EC expression of PECAM-1 and CD99 are not increased by inflammatory cytokines. However, ECs sequester these molecules in perijunctional vesicles denoted as the lateral border recycling compartment. Attachment of leukocytes at or near the junctions induces the EC to bring PECAM-1 and CD99 to the surface of the junction or to the membrane of transcellular channels that form near the junction, lining the path through which the leukocyte traverses the EC monolayer. The signal transmitted to the ECs that leads to increased surface expression of PECAM-1 and CD99 may be clustering of molecules, such as ICAM-1.

Once through the EC layer, the leukocyte still must traverse the PC layer and the BM in which the PCs are embedded. PCs also respond to inflammatory cytokines, but their levels of induced expression of adhesion molecules, largely restricted to ICAM-1 and VCAM-1, are less than on ECs (Ayres-Sander et al. 2013). Consequently, cultured PC monolayers support only limited transit of neutrophils, although neutrophils that traverse an EC monolayer become altered in an unspecified manner that increases their ability to traverse PC monolayers. PCs do express CD99, but not PECAM-1, and CD99 may also be involved in traversing the PC layer of the venular wall. In addition, PCs are contractile cells that anchor to BM proteins and can manipulate the organization of the BM; PCs also steer ex- travasating leukocytes to regions in which the $\mathrm{BM}$ is more attenuated, known as "low expression regions" (Nourshargh et al. 2010). However, the detailed functions of PCs in the process of leukocyte extravasation are less well understood than those of ECs.

During inflammation, plasma protein and leukocyte extravasation both occur at the same sites. The extravasation of leukocytes through an EC monolayer in vitro can occur without inducing inter-EC gaps that result in increased paracellular leak of macromolecules (Huang et al. 1988). The increased paracellular leak in vivo probably results directly from inflammatory cytokine exposure rather than through gaps opened by leukocytes. The extravasated plasma proteins form a provisional matrix within the tissue that can support attachment, survival, and migration of subsequently extravasated leukocytes. In cell culture models, EC leaks in response to IL-1 or TNF occur in two stages: an early but transient leak that may be mediated by cytoskeletal contraction induced by activation of a small G protein, likely Arf6 (Zhu et al. 2012), and a later, more pronounced and sustained leak dependent on new protein synthesis (Clark et al. 2007). The newly synthesized protein $(\mathrm{s})$ responsible for increased leakiness have not been defined.

Entry of leukocytes into the CNS has unique features that differ from extravasation elsewhere in the body (Man et al. 2007). Within the CNS, ECs form numerous intercellular tight junctions, the PC to EC ratio reaches 1:1, the highest in the body, and astrocyte and glial foot processes abut on the EC/PC BM, creating an additional barrier referred to as the glia limitans. As a result, resting CNS microvessels effectively prevent all protein transit, creating the "bloodbrain barrier." It is unknown to what extent these features extend to the postcapillary venules of the CNS, but basal trafficking of leukocytes is very low. Inflammation may disrupt the blood-brain barrier and passage of leukocytes through the vessel wall seems to be particularly dependent on leukocyte integrin VLA-4 (capable of recognizing cellular fibronectin as well as VCAM-1). Low-affinity VLA-4 interactions with VCAM-1 can support leukocyte tethering and 
rolling, possibly explaining why knockout of selectins has little effect on entry of leukocytes into the CNS in mouse models of neuroinflammation. LFA-1 is also important for entry into the CNS, and it may engage ICAM-2, a constitutively expressed EC molecule, as well as ICAM-1 during crawling to the EC junctions. LFA-1 may also engage JAM-A, a tight junction protein during transendothelial migration. Those cells that do get through the EC/PC/ $\mathrm{BM}$ barrier may still be prevented from entering the CNS because of the glia limitans, accumulating in the so-called Virchow-Robin space adjacent to the blood vessels. Leukocytes may also bypass the CNS vasculature to enter into the brain by crossing the more permeable choroid plexus where blood is filtered to produce cerebrospinal fluid.

In skin as in most other tissues, inflammatory cytokines induce E-selectin and VCAM-1 on venular ECs but not adjacent capillary ECs; capillary ECs are responsive to these same cytokines as shown by selective up-regulation ICAM-1 expression (Enis et al. 2005). This difference accounts for why leukocytes extravasate through venules and generally not capillaries, but the basis of this restriction of the response to cytokines is unknown. Possible contributors are differences in the shear stress detected by the ECs (high in capillaries, low in venules) and differences in BM composition. Application of shear stress to cultured microvascular ECs induces expression of the transcription factor KLF-4, which limits cytokine-induced adhesion molecule expression (Clark et al. 2011). As noted previously, the venular BM in skin microvessels has a distinct appearance by transmission electron microscopy from that of capillaries and in psoriatic lesions, when capillary loops in the dermal papillae are remodeled to become venules, these microvessels change the appearance of their BM concomitantly with the capacity of their ECs to express E-selectin and VCAM-1 (Petzelbauer et al. 1994). These data are consistent with the hypothesis that attachment to BM of different composition or organization can influence EC responses to cytokines. It should be noted that there are exceptions to the primary role of venules for extravasation. For example, in the lung, leukocytes exit into the bronchial wall via venules of the bronchial circulation but enter into the alveolus via alveolar capillaries of the pulmonary circulation. The capillary tufts within the renal glomeruli and the sinusoidal capillaries of the liver can also express adhesion molecules and support leukocyte extravasation.

The various stimuli that evoke inflammation may elicit inflammatory infiltrates that are enriched for particular types of leukocytes and these may change over time. Such selectivity and its evolution can often be explained by changes in the adhesion molecules and chemokines displayed on the EC surface. For example, E-selectin is more rapidly synthesized and displayed than is VCAM-1 or ICAM-1, but its expression on the cell surface is generally more transient, peaking at $4-6 \mathrm{~h}$ and falling to low levels by $24 \mathrm{~h}$, and better correlates with neutrophil than mononuclear cell capture and recruitment, perhaps because mononuclear cells are more adept at tethering and rolling on VCAM-1. IL-4 augments VCAM-1 expression by EC and also causes ECs to synthesize eotaxin-3, a chemokine that favors recruitment of eosinophils. Thus, the combination of TNF plus IL-4 can lead to inflammatory infiltrates that are enriched for this cell type (Briscoe et al. 1992). This kind of "specialized" inflammation has historically been associated with adaptive immunity in which $\mathrm{CD}^{+}{ }^{+}$, Th-1, Th-2, and, more recently, Th-17 cells favor their own recruitment and specific types of effector cells and, hence, are outside the scope of this review. However, it has recently been appreciated that other cell types, such as mast cells, basophils, and innate lymphocytes, also may display polarized cytokine profiles and elicit particular inflammatory patterns that are independent of antigen (Bochner and Schleimer 2001). It is likely that ECs will play a role in shaping the nature of these types of inflammatory reactions, although little is known to date what signals are involved. IL-17, for example, has little effect on ECs by itself but may modulate other cytokine responses (Bernink et al. 2013). Interestingly, IL-17 does have direct effects on PC production of chemokines, similar to previous observations using cultured 
SMCs (Eid et al. 2009), and a more integrated view of venular activation rather than EC activation may be required to understand how inflammatory reactions evolve and differentiate.

\section{VASCULAR DYSFUNCTION: FLOW DYSREGULATION, THROMBOSIS, AND CAPILLARY LEAK}

As we noted earlier in this article, changes in ECs induced by vasoactive autacoids (type I activation) and inflammatory cytokines (type II activation) contribute to inflammation. Because resting ECs actively resist the development of inflammation, these responses may be seen as interfering with normal (basal) EC function. Alternatively, such changes can be viewed as being adaptive because local inflammation is an important mechanism of host defense and homeostasis. However, there are changes that occur in ECs that disrupt homeostasis without beneficial effect to the host. Some of these are simply exaggerated or inappropriate forms of activation, whereas others result from injury and have been linked together under the category of "endothelial dysfunction" (Pober et al. 2009). As discussed above, tissue perfusion is controlled by SMC tone in the terminal arterioles. SMCs normally respond to EC-derived signals, neural signals, and humoral (hormonal) signals. Perfusion to a specific tissue can be inappropriately diminished when arteriolar SMCs in the microvasculature of a particular tissue become refractory to vasodilatory signals and/or because EC-derived vasodilatory signals are decreased. A common pattern of EC dysfunction occurs when EC lose the capacity to synthesize NO because of diminished expression of eNOS owing to TNF-mediated destabilization of its mRNA mediated by TNF-induced miR155 (Sun et al. 2012). Additionally, oxidative stress associated with inflammation can turn eNOS into a generator of superoxide anion, a change called "eNOS uncoupling" thereby reducing NO bioactivity independent of changes in eNOS mRNA or protein levels (Forstermann and Sessa 2012). TNF may also cause ECs to increase synthesis of the vasoconstrictor peptide endothelin-1 (Marsden and Brenner 1992). Paradoxically, because flow is regulated by blood pressure in addition to vascular resistance, a global decrease in blood pressure caused by widespread vasodilation throughout the circulatory system, as occurs in septic shock, may also lead to hypoperfusion. A possible explanation is that TNF increases production of PGI2 by SMCs, as well as ECs, possibly through induction of cyclo-oxygenase 2 (Jimenez et al. 2005).

Thrombosis is another potential example of EC dysfunction (Pober et al. 2009). Local intravascular coagulation may be a means of preventing hematogenous dissemination of an infection and thus part of innate immunity. However, thrombosis can also produce tissue infarcts and, when widespread, paradoxical bleeding caused by consumption of clotting factors (Esmon 2004). ECs may contribute to this by shedding microparticles, for instance plasma membrane-derived vesicles with surface-exposed PS and thus can serve as a platform for assembly of coagulation factors. TNF-activated ECs may also lose expression of thrombomodulin through mRNA destabilization and may lose their anticoagulant heparin sulfates through cytokine-induced enzymatic degradation. Tissue factor pathway inhibitor may also be down-regulated at the same time that tissue factor is synthesized and "de-encrypted," the latter process describing transfer to a location, such as to shed microparticles, where it may encounter and catalytically enhance factor VIIa. The net effect of these changes is to enhance thrombin generation and to allow thrombin to cleave fibrinogen to fibrin. At the same time, ECs or other vascular cells may enhance production of plasminogen activator inhibitors, reducing the local capacity of ECs to activate plasminogen to plas$\mathrm{min}$, and lyse fibrin thrombi as they are formed.

We noted earlier that venular leak of plasma proteins plays an important role in the inflammatory process, providing the components of a provisional matrix to support extravasating leukocytes. We also noted that, in most tissues, capillaries form a much larger surface for exchange of nutrients and wastes between tissues and blood. Under normal circumstances, capillaries (and arterioles) do not leak as a part of the 
inflammatory process. This difference may relate to the observation that capillaries (and arterioles) form tight junctions between adjacent ECs, whereas venules do not (Simionescu et al. 1975). The process of disassembly of tight junctions may be viewed as pathological and as an example of dysfunction. Capillary leak is a characteristic feature of sepsis and its development is one of the causes of organ failure in that syndrome (Gustot 2011). The mechanisms by which mediators of sepsis open tight junctions is unknown and may be distinct from the process by which venular adherens junctions are disassembled. Capillary leak may also result from EC injury and death (Joris et al. 1990).

\section{ANGIOGENESIS AND THE VASCULAR SYSTEM IN CHRONIC INFLAMMATION}

Mononuclear leukocytes (monocytes) are recruited to sites of inflammation where they may differentiate into macrophages and promote angiogenesis. Angiogenesis is defined as the migration and proliferation of ECs lining venules into surrounding tissue resulting in the formation of a capillary plexus. The recruitment of macrophages to sites of inflammation is critical for the resolution of inflammation. However, if the signal inducing the acute inflammatory response is not eradicated, chronic inflammation may ensue, and there is evidence that the transition from acute to chronic inflammation relies on an angiogenic response as a means to provide blood supply to inflamed neotissue. In this context, sustained angiogenesis may amplify the extent of macrophage infiltration and edema, and worsen tissue damage. Indeed, strategies aimed at inhibiting macrophage subsets and/or angiogenesis can reduce the degree of inflammation in several preclinical model systems. Vascular endothelial cell growth factor-A (VEGFA) and TNF derived from macrophages are potent angiogenic factors and inducers of inflammation-driven vascular remodeling in a variety of inflammatory diseases, such as psoriasis, rheumatoid arthritis, inflammatory bowel disease, and asthma. Both VEGF and TNF are produced by activated macrophages and bind to their cognate receptors on endothelium to stimulate angiogenesis. VEGF binds to VEGF receptor 2 (VEGFR2) promoting angiogenesis (Baer et al. 2013), whereas TNF binds to TNFR2, which is induced on ECs during the early stages of inflammation and mediates its proangiogenic function (Luo et al. 2006). Moreover, TNF can prime endothelium by up-regulating VEGF receptor 2 (Sainson et al. 2008). Additional inflammatory mediators can also promote angiogenesis include IL-1, IL-6, IL-17, and IL-18, and there is substantial cross talk between these cytokines and VEGF/TNF signaling pathways (Huggenberger and Detmar 2011). Chemokines play an important role both as agents that recruit macrophages and as agents produced by macrophages (or by other cells, including ECs and PCs) that may then promote or inhibit angiogenesis (Owen and Mohamadzadeh 2013).

\section{CONCLUDING REMARKS}

The blood vascular system has evolved to control tissue homeostasis. Under normal conditions, the vasculature maintains a quiescent interface between blood and tissue. On encountering an inflammatory insult, the endothelium actively participates in controlling blood flow, permeability, leukocyte infiltration, and tissue edema, changes that serve to eradicate the initial stimulus. PCs and SMCs also participate in these processes, but their roles are less well understood than those of ECs. If the stimulus persists, inflammation evolves into a chronic phase. Sustained activation of the endothelium caused by persistent inflammation can promote macrophage recruitment and neotissue angiogenesis to sustain blood flow. Understanding the vascular changes that occur during the transition from acute to chronic inflammation is crucial for our development of novel therapeutic approaches associated with debilitating inflammatory diseases.

\section{REFERENCES}

Aird WC. 2007a. Phenotypic heterogeneity of the endothelium: I. Structure, function, and mechanisms. Circ Res 100: $158-173$. 
Aird WC. 2007b. Phenotypic heterogeneity of the endothelium: II. Representative vascular beds. Circ Res 100: 174190.

Alitalo K. 2011. The lymphatic vasculature in disease. Nat Med 17: 1371-1380.

Al-Lamki RS, Wang J, Vandenabeele P, Bradley JA, Thiru S, Luo D, Min W, Pober JS, Bradley JR. 2005. TNFR1- and TNFR2-mediated signaling pathways in human kidney are cell type-specific and differentially contribute to renal injury. FASEB J 19: 1637-1645.

Armulik A, Genove G, Betsholtz C. 2011. Pericytes: Developmental, physiological, and pathological perspectives, problems, and promises. Dev Cell 21: 193-215.

Ayres-Sander CE, Lauridsen H, Maier CL, Sava P, Pober JS, Gonzalez AL. 2013. Transendothelial migration enables subsequent transmigration of neutrophils through underlying pericytes. PLoS ONE 8: e60025.

Baer C, Squadrito ML, Iruela-Arispe ML, De Palma M 2013. Reciprocal interactions between endothelial cells and macrophages in angiogenic vascular niches. Exp Cell Res 319: 1626-1634.

Barreiro O, Vicente-Manzanares M, Urzainqui A, Yanez-Mo M, Sanchez-Madrid F. 2004. Interactive protrusive structures during leukocyte adhesion and transendothelial migration. Front Biosci 9: 1849-1863.

Behringer EJ, Segal SS. 2012. Spreading the signal for vasodilatation: Implications for skeletal muscle blood flow control and the effects of ageing. J Physiol 590: 62776284.

Bernink J, Mjosberg J, Spits H. 2013. Th1- and Th2-like subsets of innate lymphoid cells. Immunol Rev 252: 133-138.

Bochner BS, Schleimer RP. 2001. Mast cells, basophils, and eosinophils: Distinct but overlapping pathways for recruitment. Immunol Rev 179: 5-15.

Bouwens EA, Stavenuiter F, Mosnier LO. 2013. Mechanisms of anticoagulant and cytoprotective actions of the protein $C$ pathway. J Thromb Haemost 11: 242-253.

Braverman IM. 1989. Ultrastructure and organization of the cutaneous microvasculature in normal and pathologic states. J Invest Dermatol 93: 2S-9S.

Briscoe DM, Cotran RS, Pober JS. 1992. Effects of tumor necrosis factor, lipopolysaccharide, and IL-4 on the expression of vascular cell adhesion molecule-1 in vivo. Correlation with $\mathrm{CD}^{+} \mathrm{T}$ cell infiltration. J Immunol 149: $2954-2960$.

Carman CV, Sage PT, Sciuto TE, de la Fuente MA, Geha RS Ochs HD, Dvorak HF, Dvorak AM, Springer TA. 2007. Transcellular diapedesis is initiated by invasive podosomes. Immunity 26: 784-797.

Clark PR, Manes TD, Pober JS, Kluger MS. 2007. Increased ICAM-1 expression causes endothelial cell leakiness, cytoskeletal reorganization and junctional alterations. J Invest Dermatol 127: 762-774.

Clark PR, Jensen TJ, Kluger MS, Morelock M, Hanidu A, Qi Z, Tatake RJ, Pober JS. 2011. MEK5 is activated by shear stress, activates ERK5 and induces KLF4 to modulate TNF responses in human dermal microvascular endothelial cells. Microcirculation 18: 102-117.

Crisan M, Yap S, Casteilla L, Chen CW, Corselli M, Park TS, Andriolo G, Sun B, Zheng B, Zhang L, et al. 2008. A perivascular origin for mesenchymal stem cells in multiple human organs. Cell Stem Cell 3: 301-313.

Dejana E, Giampietro C. 2012. Vascular endothelial-cadherin and vascular stability. Curr Opin Hematol 19: 218-223.

Dore-Duffy P, Cleary K. 2011. Morphology and properties of pericytes. Methods Mol Biol 686: 49-68.

Eid RE, Rao DA, Zhou J, Lo SF, Ranjbaran H, Gallo A, Sokol SI, Pfau S, Pober JS, Tellides G. 2009. Interleukin-17 and interferon- $\gamma$ are produced concomitantly by human coronary artery-infiltrating $\mathrm{T}$ cells and act synergistically on vascular smooth muscle cells. Circulation 119: 14241432.

Enis DR, Shepherd BR, Wang Y, Qasim A, Shanahan CM, Weissberg PL, Kashgarian M, Pober JS, Schechner JS. 2005. Induction, differentiation, and remodeling of blood vessels after transplantation of Bcl-2-transduced endothelial cells. Proc Natl Acad Sci 102: 425-430.

Esmon CT. 2004. The impact of the inflammatory response on coagulation. Thromb Res 114: 321-327.

Forstermann U, Sessa WC. 2012. Nitric oxide synthases: Regulation and function. Eur Heart $J$ 33: 829-837, 837a-837d.

Goddard LM, Iruela-Arispe ML. 2013. Cellular and molecular regulation of vascular permeability. Thromb Haemost 109: 407-415.

Gustot T. 2011. Multiple organ failure in sepsis: Prognosis and role of systemic inflammatory response. Curr Opin Crit Care 17: 153-159.

Heltianu C, Simionescu M, Simionescu N. 1982. Histamine receptors of the microvascular endothelium revealed in situ with a histamine-ferritin conjugate: Characteristic high-affinity binding sites in venules. J Cell Biol 93: 357-364.

Huang AJ, Furie MB, Nicholson SC, Fischbarg J, Liebovitch LS, Silverstein SC. 1988. Effects of human neutrophil chemotaxis across human endothelial cell monolayers on the permeability of these monolayers to ions and macromolecules. J Cell Physiol 135: 355-366.

Huggenberger R, Detmar M. 2011. The cutaneous vascular system in chronic skin inflammation. J Investig Dermatol Symp Proc 15: 24-32.

Imaizumi T, Yoshida H, Satoh K. 2004. Regulation of CX3CL1/fractalkine expression in endothelial cells. $J$ Atheroscler Thromb 11: 15-21.

Jimenez R, Belcher E, Sriskandan S, Lucas R, McMaster S, Vojnovic I, Warner TD, Mitchell JA. 2005. Role of Tolllike receptors 2 and 4 in the induction of cyclooxygenase-2 in vascular smooth muscle. Proc Natl Acad Sci 102: 4637-4642.

Joris I, Cuenoud HF, Doern GV, Underwood JM, Majno G. 1990. Capillary leakage in inflammation. A study by vascular labeling. Am J Pathol 137: 1353-1363.

Ley K, Laudanna C, Cybulsky MI, Nourshargh S. 2007. Getting to the site of inflammation: The leukocyte adhesion cascade updated. Nat Rev Immunol 7: 678-689.

Lorant DE, Patel KD, McIntyre TM, McEver RP, Prescott SM, Zimmerman GA. 1991. Coexpression of GMP-140 and PAF by endothelium stimulated by histamine or thrombin: A juxtacrine system for adhesion and activation of neutrophils. J Cell Biol 115: 223-234. 
Luo D, Luo Y, He Y, Zhang H, Zhang R, Li X, Dobrucki WL, Sinusas AJ, Sessa WC, Min W. 2006. Differential functions of tumor necrosis factor receptor 1 and 2 signaling in ischemia-mediated arteriogenesis and angiogenesis. Am J Pathol 169: 1886-1898.

Majesky MW. 2007. Developmental basis of vascular smooth muscle diversity. Arterioscler Thromb Vasc Biol 27: $1248-1258$.

Majno G, Palade GE, Schoefl GI. 1961. Studies on inflammation: II. The site of action of histamine and serotonin along the vascular tree: A topographic study. J Biophys Biochem Cytol 11: 607-626.

Man S, Ubogu EE, Ransohoff RM. 2007. Inflammatory cell migration into the central nervous system: A few new twists on an old tale. Brain Pathol 17: 243-250.

Marsden PA, Brenner BM. 1992. Transcriptional regulation of the endothelin-1 gene by TNF- $\alpha$. Am J Physiol 262: C854-C861.

Mortier A, Van Damme J, Proost P. 2012. Overview of the mechanisms regulating chemokine activity and availability. Immunol Lett 145: 2-9.

Muller WA. 2011. Mechanisms of leukocyte transendothelial migration. Annu Rev Pathol 6: 323-344.

Nourshargh S, Hordijk PL, Sixt M. 2010. Breaching multiple barriers: Leukocyte motility through venular walls and the interstitium. Nat Rev Mol Cell Biol 11: 366-378.

Opitz B, Hippenstiel S, Eitel J, Suttorp N. 2007. Extra- and intracellular innate immune recognition in endothelial cells. Thromb Haemost 98: 319-326.

Owen JL, Mohamadzadeh M. 2013. Macrophages and chemokines as mediators of angiogenesis. Front Physiol 4 159.

Pan J, Xia L, McEver RP. 1998. Comparison of promoters for the murine and human P-selectin genes suggests speciesspecific and conserved mechanisms for transcriptional regulation in endothelial cells. J Biol Chem 273: $10058-$ 10067.

Payne GW, Madri JA, Sessa WC, Segal SS. 2003. Abolition of arteriolar dilation but not constriction to histamine in cremaster muscle of $\mathrm{eNOS}^{-1-}$ mice. Am J Physiol Heart Circ Physiol 285: H493-H498.

Petzelbauer P, Pober JS, Keh A, Braverman IM. 1994. Inducibility and expression of microvascular endothelial adhesion molecules in lesional, perilesional, and uninvolved skin of psoriatic patients. J Invest Dermatol 103: 300-305.
Pober JS, Sessa WC. 2007. Evolving functions of endothelial cells in inflammation. Nat Rev Immunol 7: 803-815.

Pober JS, Tellides G. 2012. Participation of blood vessel cells in human adaptive immune responses. Trends Immunol 33: $49-57$.

Pober JS, Min W, Bradley JR. 2009. Mechanisms of endothelial dysfunction, injury, and death. Annu Rev Pathol 4: 71-95.

Rondaij MG, Bierings R, Kragt A, van Mourik JA, Voorberg J. 2006. Dynamics and plasticity of Weibel-Palade bodies in endothelial cells. Arterioscler Thromb Vasc Biol 26: 1002-1007.

Rous P, Smith F. 1931. The gradient of vascular permeability: III. The gradient along the capillaries and venules of frog skin. J Exp Med 53: 219-242.

Sainson RC, Johnston DA, Chu HC, Holderfield MT, Nakatsu MN, Crampton SP, Davis J, Conn E, Hughes CC. 2008. TNF primes endothelial cells for angiogenic sprouting by inducing a tip cell phenotype. Blood 111: 4997-5007.

Simionescu M, Simionescu N, Palade GE. 1975. Segmental differentiations of cell junctions in the vascular endothelium. The microvasculature. J Cell Biol 67: 863-885.

Suarez Y, Wang C, Manes TD, Pober JS. 2010. Cutting edge: TNF-induced microRNAs regulate TNF-induced expression of E-selectin and intercellular adhesion molecule-1 on human endothelial cells: Feedback control of inflammation. J Immunol 184: 21-25.

Sun HX, Zeng DY, Li RT, Pang RP, Yang H, Hu YL, Zhang Q, Jiang Y, Huang LY, Tang YB, et al. 2012. Essential role of microRNA-155 in regulating endothelium-dependent vasorelaxation by targeting endothelial nitric oxide synthase. Hypertension 60: 1407-1414.

Zavoico GB, Ewenstein BM, Schafer AI, Pober JS. 1989. IL-1 and related cytokines enhance thrombin-stimulated PGI2 production in cultured endothelial cells without affecting thrombin-stimulated von Willebrand factor secretion or platelet-activating factor biosynthesis. J Immunol 142: 3993-3999.

Zhu W, London NR, Gibson CC, Davis CT, Tong Z, Sorensen LK, Shi DS, Guo J, Smith MC, Grossmann AH, et al. 2012. Interleukin receptor activates a MYD88-ARNOARF6 cascade to disrupt vascular stability. Nature 492: $252-255$. 


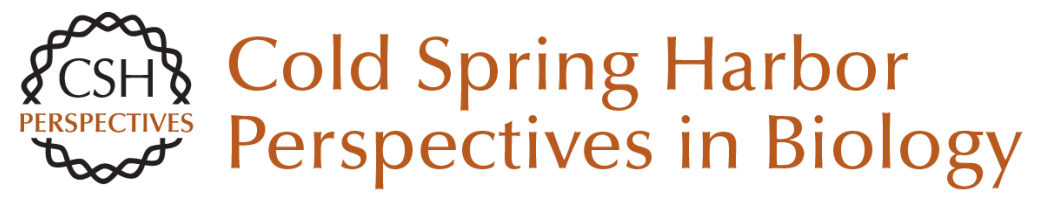

\section{Inflammation and the Blood Microvascular System}

Jordan S. Pober and William C. Sessa

Cold Spring Harb Perspect Biol 2015; doi: 10.1101/cshperspect.a016345 originally published online October 23, 2014

Subject Collection Innate Immunity and Inflammation

Group 2 Innate Lymphoid Cells in Health and Disease

Brian S. Kim and David Artis

Inflammation and the Blood Microvascular

System

Jordan S. Pober and William C. Sessa

Sinusoidal Immunity: Macrophages at the

Lymphohematopoietic Interface

Siamon Gordon, Annette Plüddemann and

Subhankar Mukhopadhyay

Allergic Inflammation--Innately Homeostatic Laurence E. Cheng and Richard M. Locksley

Approaching the Next Revolution? Evolutionary Integration of Neural and Immune Pathogen

Sensing and Response

Kevin J. Tracey

Inflammasomes

Marcel R. de Zoete, Noah W. Palm, Shu Zhu, et al.

IL-6 in Inflammation, Immunity, and Disease

Toshio Tanaka, Masashi Narazaki and Tadamitsu Kishimoto
The Chemokine System in Innate Immunity Caroline L. Sokol and Andrew D. Luster

Microbial Sensing by Toll-Like Receptors and Intracellular Nucleic Acid Sensors Surya Pandey, Taro Kawai and Shizuo Akira

Tumor Necrosis Factor Superfamily in Innate Immunity and Inflammation John Sedý, Vasileios Bekiaris and Carl F. Ware

Emerging Principles Governing Signal Transduction by Pattern-Recognition Receptors Jonathan C. Kagan and Gregory M. Barton

Lipid Mediators in the Resolution of Inflammation Charles N. Serhan, Nan Chiang, Jesmond Dalli, et al.

Transcriptional Control of Inflammatory

Responses

Stephen T. Smale and Gioacchino Natoli

DNA Degradation and Its Defects

Kohki Kawane, Kou Motani and Shigekazu Nagata

For additional articles in this collection, see http://cshperspectives.cshlp.org/cgi/collection/

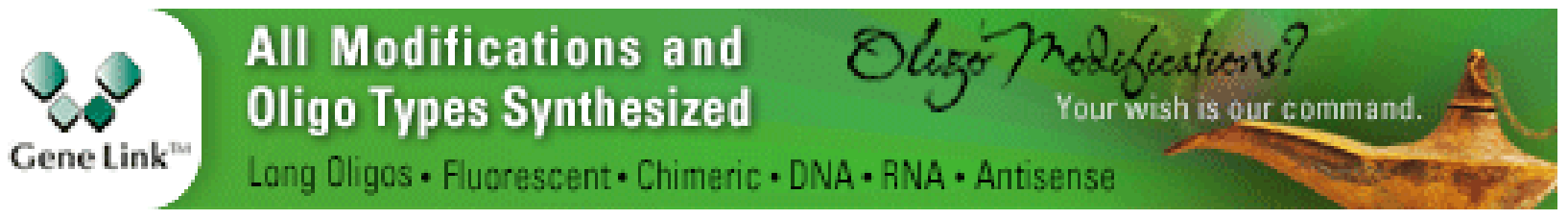

Copyright @ 2015 Cold Spring Harbor Laboratory Press; all rights reserved 